\title{
Study on Defects Edge Detection in Infrared Thermal Image Based on Ant Colony Algorithm
}

\author{
TANG Qingju ${ }^{\mathrm{a}, \mathrm{b}}$, DAI Jingmin ${ }^{\mathrm{a}}$, LIU Chunsheng ${ }^{\mathrm{b}}$, LIU Yuanlin ${ }^{\mathrm{b}}$ and REN \\ Chunping ${ }^{\mathrm{b}}$ \\ ${ }^{a}$ School of Electrical Engineering and Automation, Harbin Institute of \\ Technology, Harbin, 150001, P.R.China \\ ${ }^{b}$ School of Mechanical Engineering, Heilongjiang University of Science and \\ Technology, Harbin, 150022, P.R.China \\ E-mail:tangqingju@126.com
}

\begin{abstract}
Edge extraction is an important part in the detection of infrared thermal images. Ant colony algorithm has the characteristics of high efficiency, high noise suppression, and comprehensive information of edge information. The basic principle of ant colony algorithm is analyzed. An ant colony optimization algorithm for image edge detection is established. And to have defective parts for analysis of infrared thermography The ant colony algorithm and the classical Canny operator are compared and analyzed. The experimental results show that the algorithm has high efficiency, comprehensive information and high computational efficiency.
\end{abstract}

Keywords: Infrared thermography; Defect edge detection; Ant colony algorithm

\section{Introduction}

Edge is one of the most simple and important image features, including a large amount of image information, which has been widely used in the field of image recognition, segmentation, enhancement, compression and so on.Edge exists in the target and background, target and target, region and region, is the image of the object structure, texture, shape of important information, edge detection for image post-processing and analysis provides an important parameters, the follow-up to further feature description, matching and recognition, etc. have a significant impact on [1-3]. Edge detection is the most basic technology in the field of image processing and computer vision. Its purpose is to extract the edge of the object in the image, which lays a good foundation for image fusion, shape extraction, image segmentation, image matching and image tracking [4-7]. Edge detection is a hot research topic in the field of digital image processing. The traditional edge detection method is based on the gray value of the image to determine the edge, because of the existence of various noise has a great impact on the edge detection, the existing edge detection technology needs to set many parameters, the adaptive ability is poor, it is difficult to detect more complex images, so image edge detection technology needs to be further improved and developed. The commonly used edge detection method is based on the gray level of the image, and the edge detection method has many kinds, such as Log edge detection operator, Canny edge detection operator, Roberts edge detection operator. The classical edge detection method is to calculate the gradient amplitude information of the image, and judge whether the image is edge. However, these algorithms are easy to be affected by the noise. When the gray level of image edge is not obvious, it is easy to cause the image edge detection [8-11].

In recent years, with various new algorithms and artificial intelligence theory, some new edge detection methods, such as wavelet transform and wavelet packet edge 
detection, edge detection algorithm based on mathematical morphology [12-15], fuzzy theory and neural network edge detection, edge detection using genetic algorithm, etc. [16-18].

In addition, ant colony algorithm is applied to image edge detection in recent years because of its advantages, such as high computational speed, high efficiency of noise reduction, complete extraction of edge information.

\section{Ant Colony Algorithm}

Ant colony algorithm is a kind of optimization algorithm proposed by Italy scholar M Dorigo in 1992. It is a kind of random search method with advantages of discrete, parallel and robust [19-21]. Ant colony algorithm is a kind of simulated evolutionary algorithm, which is based on the behavior of ants in searching for food. Ants in their own path through the path to release a substance called pheromone to carry out information, according to the intensity of the pheromone to guide their forward direction; the more the more ants the path of its pheromone is stronger, and the information will gradually over time and gradually volatile. Therefore, the probability of the path of the ant is greater, and the ant colony is able to adapt to the environment, and when the ant colony of the movement path of the obstacle, they can quickly find an optimal path, the whole ant colony will find the optimal path. Ant colony optimization algorithm is the mechanism through which the positive feedback, after many iterations, the optimal solution is obtained. In the ant path structure, the ant search behavior is influenced by the intensity of pheromone and the heuristic information. The ant colony search for the shortest path to the source of food. The discrete and parallel characteristics of ant colony algorithm has certain advantages in processing digital images. The genetic algorithm flow chart is shown in Figure. 1.

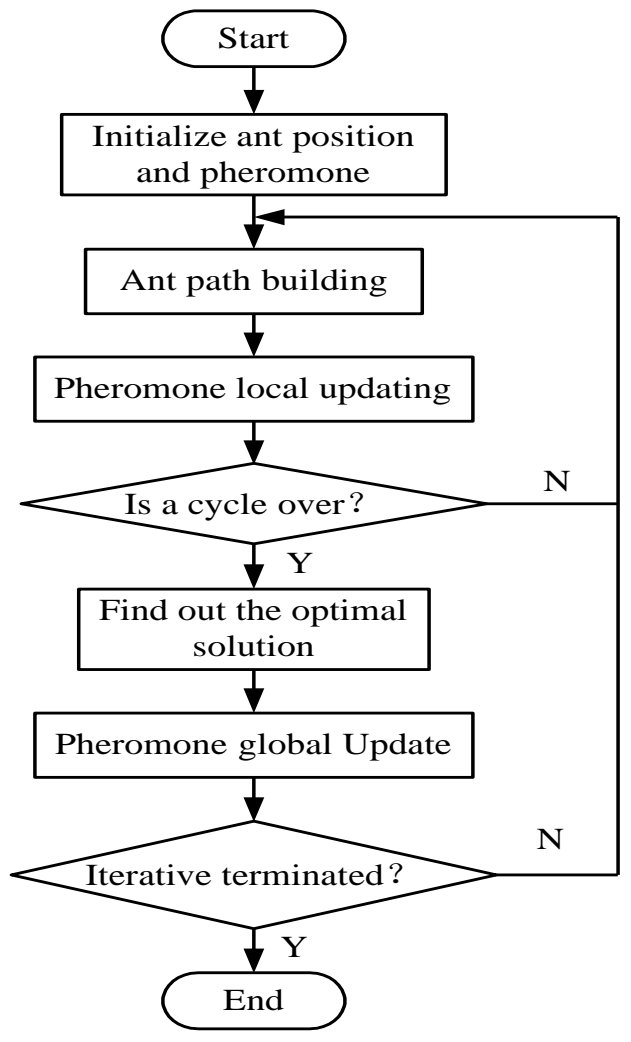

Figure 1. Genetic Algorithm Flow Chart 


\section{Image Edge Detection Algorithm Based on Ant Colony Optimization}

The principle of ant colony algorithm is that the image is abstracted into an undirected graph, and then the ant colony ant colony is randomly placed in the graph. The ants will choose one target location as the next target. The more attractive the ant colony is, the higher the value of the edge points. The edge of the image can be obtained by analyzing the distribution of the pheromone intensity.

\subsection{Initial Ant Distribution}

In general, the number of ants is set to $K=\sqrt{M \times N}$ ( $M$ and $N$ denonte the length and width of the picture respectively).

\subsection{Transfer Probability}

The probability that the ants from the pixel $(i, j)$ to the pixel $(k, l)$ is

$P(k, l)=\frac{\tau_{(k, l)}^{\alpha} \eta_{(k, l)}^{\beta} w_{(k, l)}}{\sum_{j \in \Omega} \tau_{(k, l)}^{\alpha} \eta_{(k, l)}^{\beta} w_{(k, l)}},(k, l) \in \Omega$

\subsection{Transfer rule}

Ants move from pixels to the rules of the pixels.

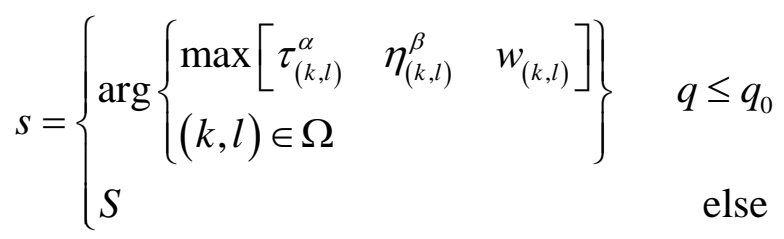

Where, $q$ is a random and $q \in[0,1], q_{0}$ is a preset value; $S$ is a selected random.

\subsection{Pheromone Update}

When ants move from pixel $(i, j)$ to pixel $(k, l)$, the pheromone updates rule is as follows

$$
\left\{\begin{array}{l}
\tau_{(k, l)}=(1-\rho) \cdot \tau_{(k, l)}+\rho \Delta \tau_{(k, l)} \\
\Delta \tau_{(k, l)}=\eta_{(k, l)}
\end{array}\right.
$$

Where, $\rho \in(0,1)$ indicates that pheromone volatile factor.

\subsection{Edge Extraction}

After the completion of the set of iterations, the choice of a threshold, according to the intensity of each pixel of the pheromone intensity to determine whether the point is the edge point 


\section{Experimental Simulation and Analysis}

\subsection{Experimental System}

SiC coated high-temperature alloy plates with defects was detected using pulsed thermographic system. Pulsed infrared thermography is a nondestructive testing technique based on the theory of heat transfer and infrared imaging theory. In the actual detection, there are two different forms of reflection and transmission methods, which are shown in Figure. 1.When the infrared camera and excitation source are in the same side of the tested component, it is called reflection detection method; When the infrared camera and excitation source are in the different sides of the tested component, it is called transmission detection method.

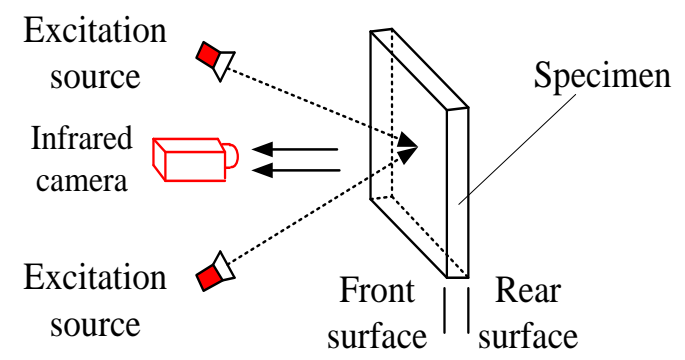

(a) Reflection Method

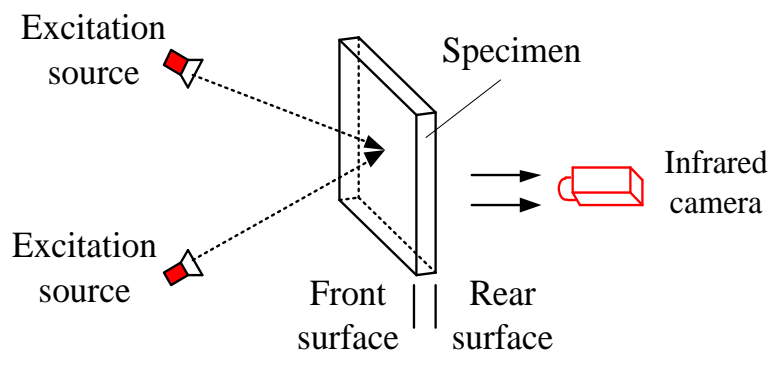

(b) Transmission Method

Figure 1. Reflection and Transmission Method

As the $\mathrm{SiC}$ coated high-temperature alloy plate with good heat resistance and thinner thickness, the reflection detection method is suitable. SiC coated hightemperature alloy plates with defects was detected using pulsed thermographic system, and the principle of the system is shown in Figure .2. The infrared thermal imaging system is the key device for infrared thermal wave nondestructive detection. It receives the infrared light from the target and the surrounding background using optical system, and converts it into electrical signal by the detecting element. Infrared thermal imaging system mainly refers to the infrared camera. It conjugates the measured object's infrared radiation into visual visible image, and display in the form of intuitive thermal image. The temperature signal of the component surface is collected by the infrared camera SC7000 produced by FLIR company, which is shown in Figure. 3.. The infrared camera has the advantages of high sensitivity and short response time. The working band of SC7000 is 3.1-5.6 m, with a resolution of $320 \times 256$ pixels, the maximum full frame rate up to $170 \mathrm{~F} / \mathrm{s}$. The equivalent noise temperature difference at room temperature is $20 \mathrm{mK}$. 


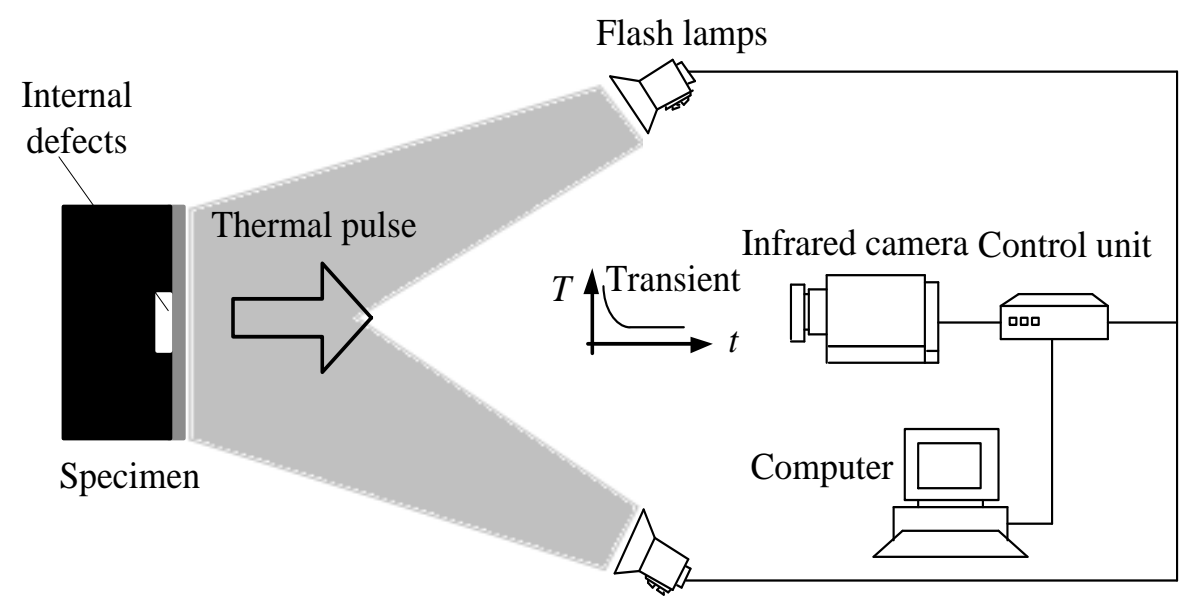

Figure. 2 Principle of Pulsed Thermographic System
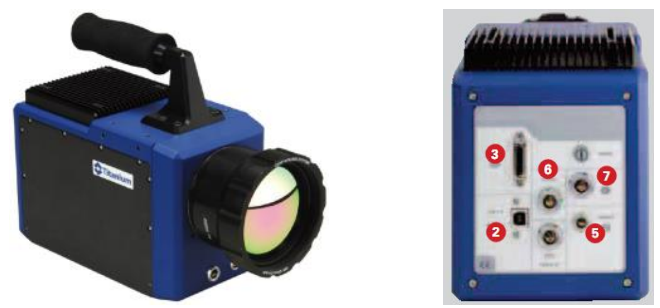

Figure. 3 Infrared Camera SC7000

Heat one side of the component using flash lamps. If a defect or discontinuity exists in the component, there will be a difference in the surface temperature of the component between defective and other regions. The image sequence to record the surface temperature distribution of the component can be captured and obtained by using an infrared camera. And then the image sequence will be processed by the signal processing algorithm, thus according to the difference between the different regions in the image, the specific information of the defects in the components can be realized.

\subsection{Specimen}

Figure. 4 and Table. 1 shows the the structure size and defect distribution of the specimen. Figure .5 shows the photo of the specimen. The coating was prepared by plasma spraying, and the prefabricated defects were flat blind holes in the hightemperature alloy substrate. 


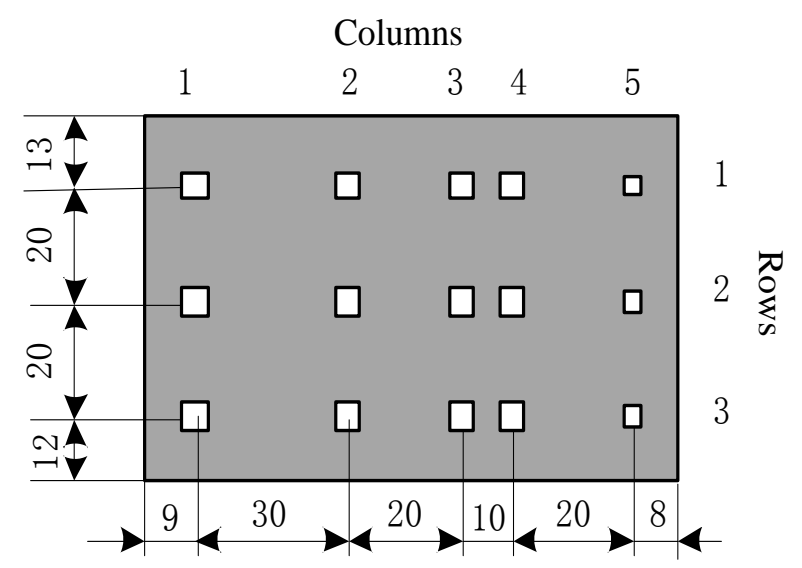

Figure. 4 SiC Coated High Temperature Superalloy Substrate with Flat Square Blind Hole Defects

Table 1. Defects Size of SiC Coated High Temperature Superalloy Substrate

\begin{tabular}{c|ccccc}
\hline Col & 1 & 2 & 3 & 4 & 5 \\
\hline \multirow{2}{*}{1} & $\mathrm{DL}=5.5$ & $\mathrm{DL}=5.5$ & $\mathrm{DL}=5.5$ & $\mathrm{DL}=5.5$ & $\mathrm{DL}=4.5$ \\
& $\mathrm{DH}=0.5$ & $\mathrm{DH}=0.5$ & $\mathrm{DH}=0.5$ & $\mathrm{DH}=0.5$ & $\mathrm{DH}=0.5$ \\
2 & $\mathrm{DL}=5.5$ & $\mathrm{DL}=5.5$ & $\mathrm{DL}=5.5$ & $\mathrm{DL}=5.5$ & $\mathrm{DL}=4.5$ \\
& $\mathrm{DH}=0.4$ & $\mathrm{DH}=0.4$ & $\mathrm{DH}=0.4$ & $\mathrm{DH}=0.4$ & $\mathrm{DH}=0.4$ \\
3 & $\mathrm{DL}=5.5$ & $\mathrm{DL}=5.5$ & $\mathrm{DL}=5.5$ & $\mathrm{DL}=5.5$ & $\mathrm{DL}=4.5$ \\
& $\mathrm{DH}=0.3$ & $\mathrm{DH}=0.3$ & $\mathrm{DH}=0.3$ & $\mathrm{DH}=0.3$ & $\mathrm{DH}=0.3$ \\
\hline
\end{tabular}

Note: DL-the edge of defects; $\mathrm{DH}$-the depth of defects.

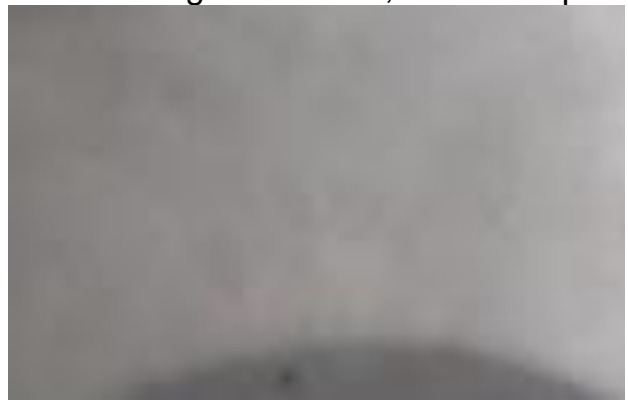

(a) Back surface of the specimen

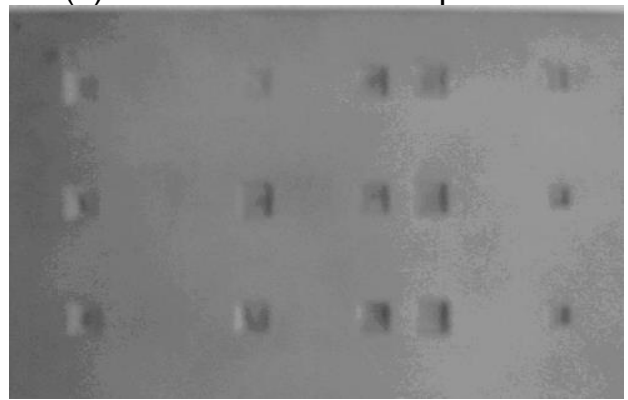

(b) Front surface of the specimen

Figure. 5 Flat blind Hole Defects in SiC Coated High Temperature Superalloy Substrate

4.3 Defects Edge Detection 
In this paper, the defects edge of infrared thermal image is extracted. Figure.6 shows a thermal image captured the infrared camera. Figure.7 shows the image after gray level transformation. Using Canny edge detection operator and ant colony algorithm to extract the edge of the image. Specific results are shown in Figure. 8 and Figure. 9.

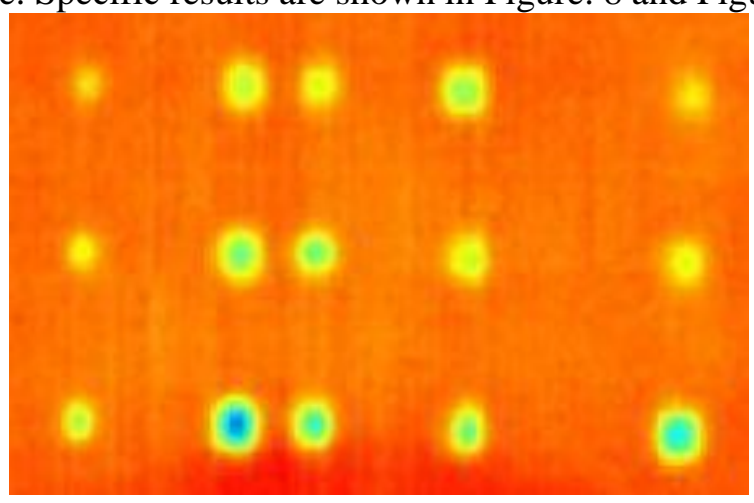

Figure.6 The Input Infrared Image

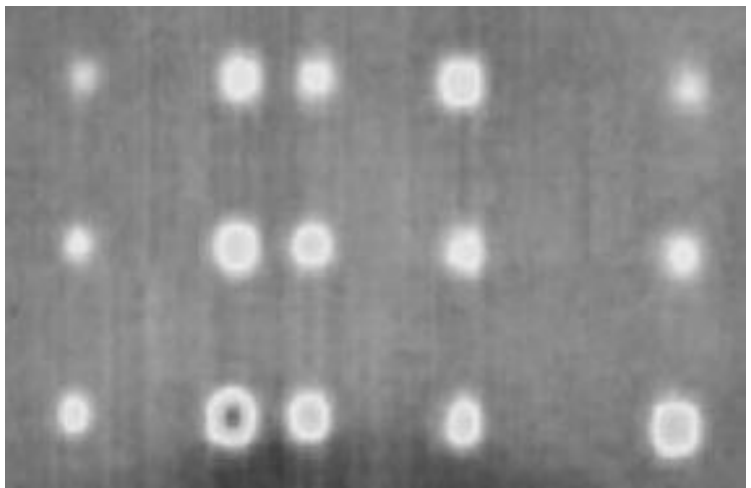

Figure.7 The Image After Gray Level Transformation

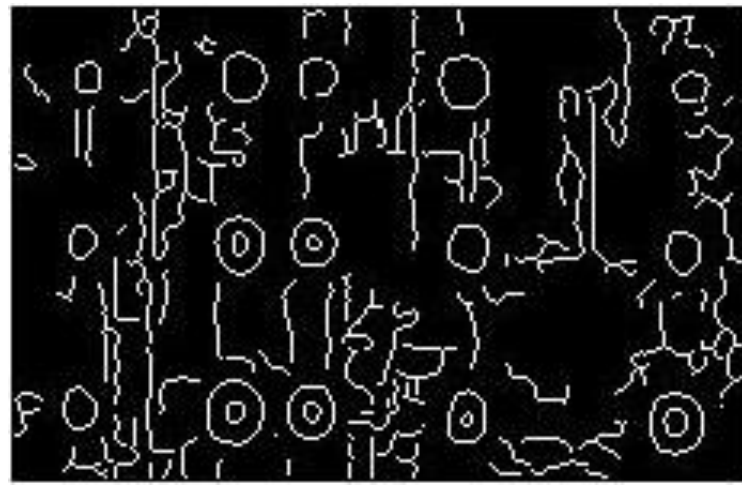

Figure.8 Processed by Canny Pperator 


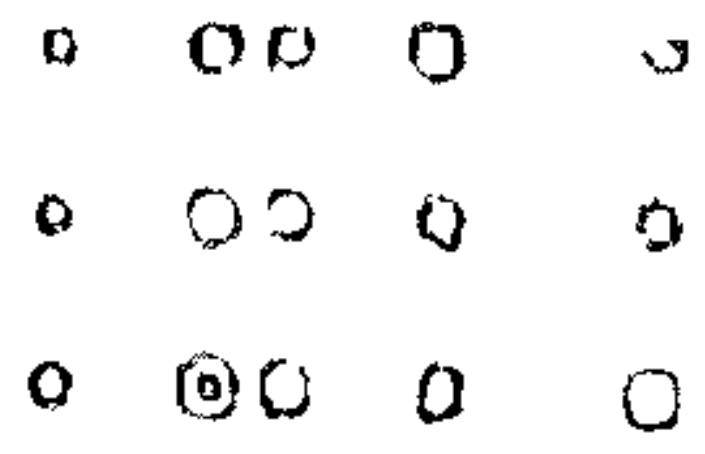

Figure.9 Processed by Ant Ant Colony Algorithm

The experimental results show that the algorithm has the characteristics of high efficiency, strong ability of restraining the noise and the edge information, and can effectively extract the real edge of the object.

\section{Conclusion}

Based on the analysis of the principle of ant colony algorithm, the image edge detection method based on ant colony algorithm is discussed. The comparison of canny and ant colony algorithm is carried out. The results show that the ant colony algorithm has the advantages of high efficiency, comprehensive accuracy of edge information in extracting the edge of infrared image.

\section{Acknowledgments}

This project is supported by Youth Innovation Talent Training Program of Heilongjiang Province Regular Institutions of Higher Education "Study on CFRP laminate defects detection using infrared thermal wave nondestructive testing technology under modulated laser exitation", Heilongjiang Province Natural Science Fund (Grant No. QC2013C054), and Cooperation Project between Heilongjiang Province and Chinese Academy of Science (Grant No. YS15A10).

\section{References}

[1] S. Mallat and Z. Sifen, "Characterizations of Signals from Multiscale Edges", J. IEEE Trans. On Pattern Analysis and Machine Intelligence, vol. 14, (1992), pp. 710-732.

[2] C. L. Molina, B. D. Baets and H. Bustince, "Multiscale edge detection based on gaussian smoothing and edge tracking", J. Knowledge Based Systems, vol. 44, (2013), pp.101-111.

[3] P. G. Nes, "Fast multi-scale edge detection in medical ultrasound signals", J. Signal Processing, vol. 92, (2012), pp. 2394-2408.

[4] J. J. Tabor, H. M. Salis and Z. B. Simpson, "A synthetic genetic edge detection program”, J. Cell, vol. 137, (2009),pp. 1272-1281.

[5] S. Vitulano, C. Di Ruberto and M. Nappi, "Edge Detection", J. International Journal of Pattern Recognition \& Artificial Intelligence, vol. 12, no. 7, (2011), pp.130.

[6] R. M.-Carnicer, F. J. M.-Cuevas and A. C.-Poyato, "On candidates selection for hysteresis thresholds in edge detection”, J. Pattern Recognition, vol. 42, (2009), pp.1284-1296.

[7] M. T. Alonso, C. López-Martínez and J. J. Malorquí, "Edge Enhancement Algorithm Based on the Wavelet Transform for Automatic Edge Detection in SAR Images”, J. Geoscience \& Remote Sensing IEEE Transactions on vol. 49, (2011), pp. 222-235.

[8] M. G. Naraghi, M. Koohi and A. Shakery, "Edge Detection in Multispectral Images Based on Structural Elements,J. International Journal of Multimedia \& Its Applications, vol. 3, (2011). 
[9] S. B. Kutty, S. Saaidin nd P. N. A. M. Yunus, "Evaluation of canny and sobel operator for logo edge detection", Technology Management and Emerging Technologies (ISTMET), Washington, America, October (2014), pp.153-156.

[10] B. H. Lang, L. Y Shen and T. L. Han, "An Adaptive Edge Detection Method Based on Canny Operator", J. Advanced Materials Research, no. 255-260, (2011), pp. 2037-2041.

[11] J. Malik, G. Sainarayanan and R Dahiya, "PERSONAL AUTHENTICATION USING PALMPRINT WITH SOBEL CODE, CANNY EDGE AND PHASE CONGRUENCY FEATURE EXTRACTION METHOD”, J.Ictact Journal on Image \& Video Processing, vol. 2, (2012).

[12] P. W. Peng, R. S. Che and D. Ye, "An illumination independent edge detection and fuzzy enhancement algorithm based on wavelet transform for non-uniform weak illumination images", J. Pattern Recognition Letters, vol. 29, (2008), pp. 192-199.

[13] Z.Y.-Qian, G.W.-Hua and C.Z.-Cheng, "Medical Images Edge Detection Based on Mathematical Morphology", Engineering in Medicine and Biology Society, 27th Annual International Conference of the IEEE, Washington, America, February (2006), pp. 6492-6495.

[14] D .Wei and W. Tong, "Research on Edge Detection Based on Mathematical Morphology Algorithm. 2010 International Conference on Optoelectronics and Image Processing IEEE Computer Society, Washington, America, March (2010), pp. 211-213.

[15] X. Bai and F. Zhou, "Edge Detection Based on Mathematical Morphology and Iterative Thresholding", J. Computational Intelligence \& Security, vol. 2, (2006), pp. 1849-1852.

[16] C. R. Mihalache and M. Craus, "Neural network and fuzzy membership functions based edge detection for digital images", System Theory, Control and Computing (ICSTCC), 2012 16th International Conference on IEEE, London, England, June 1-6 (2012).

[17] A. Jubai and B. J. J. Yang, "Combining fuzzy theory and a genetic algorithm for satellite image edge detection”, J. International Journal of Remote Sensing, vol. 27, (2006), pp. 3013-3024.

[18] T. Chaira and A. K. Ray, "A new measure using intuitionistic fuzzy set theory and its application to edge detection", J. Applied Soft Computing, vol. 8, (2008), pp. 919-927 .

[19] M. Dorigo, M. Birattari and T. Stutzle, "Ant colony optimization :artificial ants as a computational intelligence technique”, J. IEEE Computational Intelligence Magazine, vol. 11, (2006), pp. 28-39

[20] R. J. Mullen, D. N. Monekosso and P. Remagnino, "Ant algorithms for image feature extraction", J. Expert Systems With Applications, vol. 40, (2013), pp. 4315-4332.

[21] Z. Wei, P. Xiaohong and L. Zhi, "Manufacturing service scheduling strategy on cloud model ant colony optimization”, J. Computer Integrated Manufacturing Systems, (in Chinese), vol. 18, (2012), pp.201-207.

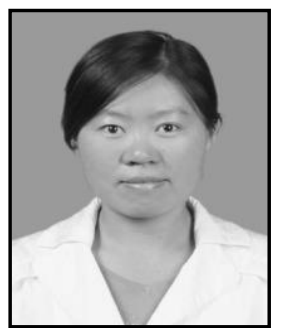

\section{Authors}

TANG Qingju received the B.S. and M.S. degrees from Harbin University of commerce, Harbin, China, in 2003 and 2006, respectively, and the Ph.D. degree from Harbin Institute of Science and Technology, Harbin, China, in 2014.She is currently a Research Engineer in School of Mechanical Engineering of Heilongjiang University of Science and Technology. At the same, she is a postdoctor of Harbin Institute of Technology. Her research interests lie in the area of infrared nondestructive testing and advanced manufacture. 
International Journal of Signal Processing, Image Processing and Pattern Recognition Vol. 9, No. 4 (2016) 